Original research article

\title{
Clinical comparison of platelet-rich plasma injection and daily celecoxib administration in the treatment of early knee osteoarthritis: A randomized clinical trial
}

\author{
Rubén Reyes-Sosa 1, \#, Agustin Lugo-Radillo 2, \# *, Maria del Rocio Ruiz-Olivera ${ }^{1}$, \\ Lizzet Cruz-Santiago ${ }^{1}$, Celia Rubí García-Cruz ${ }^{1}$, Oliver Mendoza-Cano ${ }^{3}$ \\ ${ }^{1}$ Instituto Mexicano del Seguro Social, Hospital General de Zona No. 1, Oaxaca, Mexico \\ 2 CONACYT - Universidad Autónoma Benito Juárez de Oaxaca, Faculty of Medicine and Surgery, Oaxaca, Mexico \\ ${ }^{3}$ Universidad de Colima, Faculty of Civil Engineering, Colima, Mexico
}

\begin{abstract} effectiveness of PRP against an NSAID. function were measured at baseline and at 1, 3, 6 and 12 months after the start of the treatment. age, sex or knee OA grade II or III. significant difference is independent of age, sex or knee OA grade II or III.

Keywords: Celecoxib; Inflammation; Knee; Osteoarthritis; Platelet-Rich Plasma

Highlights:

- Platelet-rich plasma (PRP) has been posited as an alternative treatment for knee OA.

- Intra-articular PRP improved VAS in comparison to celecoxib.

- PRP was better than celecoxib in improving pain, function and stiffness in knee OA.

- Differences persisted after adjusting for sex or for any grade of knee OA.

- Our results suggest that PRP is better than NSAIDs for the treatment of knee OA.
\end{abstract}

Background: Oral and topical nonsteroidal anti-inflammatory drugs (NSAIDs), analgesics and intra-articular corticosteroid injections are the recommended first line of treatment for knee osteoarthritis (OA); however, they have serious side effects. Platelet-rich plasma (PRP) has been posited as an effective and safer alternative treatment for knee OA. Hitherto, there is only one study comparing the

Aim of the study: The aim of this study was to determine the effectiveness of PRP against celecoxib in the treatment of early knee OA. Methods: 60 patients with knee OA grade II and III were randomly alocated in two groups. Group 1 received one injection of autologous PRP in each affected knee, with a reinjection after 15 days; Group 2 received $200 \mathrm{mg}$ of oral celecoxib each $24 \mathrm{~h}$ for a year. Visual Analogue Scale (VAS), total Western Ontario and McMaster Universities Arthritis Index (WOMAC) and WOMAC subscales for pain, stiffness and

Results: At the end of the study PRP was significantly better than celecoxib $(p<0.05)$ in improving VAS $(40.40 \%)$, total WOMAC (58.95\%) and WOMAC subscales of pain (50.60\%), stiffness (34.13\%) and function (51.90\%). Significant differences remained after adjusting for

Conclusions: Intra-articular PRP is significantly better than celecoxib in improving pain, function and stiffness in early knee OA. This

\section{Introduction}

Oral and topical NSAIDs, analgesics and intra-articular corticosteroid injections are the recommended first line of treatment for knee OA (Hochberg et al., 2012). These therapies are only temporal and symptomatic, and they do not modify cartilage degeneration (Laudy et al., 2015; Say et al., 2013); moreover, they are often accompanied by clinically significant side effects (Law et al., 2015; Lee et al., 2016; Simon, 2013; Solomon et al., 2017). In this regard, PRP is posited as a potential symptomatic (Laudy et al., 2015) and cartilage regeneration (Say et al., 2013) therapeutic alternative for knee OA. PRP is a plasma in which, the platelet fraction has been concentrated, usually by centrifugation (Laudy et al., 2015). It has shown, in vitro, to decrease the concentration of inflammatory mediators in cartilage and synoviocytes (Sundman et al., 2014; Wang et al., 2015) and to stimulate the synthesis of cartilage (Sakata et al., 2015; Sundman et al., 2014). PRP has also shown to produce, in vivo, a significant improvement in knee OA, regarding pain (Bottegoni et al., 2016; Kavadar et al., 2015; Kilincoglu et al., 2015; Kon et al., 2010; Montanez-Heredia et al., 2016),

\footnotetext{
* Corresponding author: Agustin Lugo-Radillo, CONACYT - Universidad Autónoma Benito Juárez de Oaxaca, Faculty of Medicine and Surgery, 68020 Oaxaca, Mexico; e-mail: alugora@conacyt.mx

\# These authors contributed equally to this work.

http://doi.org/10.32725/jab.2020.012

Submitted: 2019-01-21 • Accepted: 2020-08-18 • Prepublished online: 2020-08-20

J Appl Biomed 18/2-3: 41-45 • EISSN 1214-0287 • ISSN 1214-021X

(c) 2020 The Authors. Published by University of South Bohemia in České Budějovice, Faculty of Health and Social Sciences.

This is an open access article under the CC BY-NC-ND license.
} 
functionality (Bottegoni et al., 2016; Kavadar et al., 2015; Kilincoglu et al., 2015; Montanez-Heredia et al., 2016), inflammatory markers (Yin et al., 2016) and structural regeneration (Yin et al., 2016).

Therefore, PRP appears to be a potential clinical alternative in the treatment of knee OA; However, hitherto results have only shown a limited to moderate effect of PRP in knee OA (Laudy et al., 2015). Moreover, to the extent of our knowledge, there is only a study comparing the efficacy of PRP versus NSAIDs (Buendia-Lopez et al., 2018; Mundy, 2016). Thus, the aim of this study was to determine if PRP is significantly effective in the treatment of knee OA in comparison to celecoxib, which is the NSAID recommended for knee OA.

\section{Materials and methods}

\section{Selection and sorting of patients}

Patients attending the outpatient service of Orthopedics at the Mexican Social Security Institute's (IMSS) General Hospital 1 in Oaxaca, Mexico, whom were diagnosed with knee OA and previously treated with paracetamol without improvement, were randomly allocated by minimization in two groups. Group 1 was given $3 \mathrm{ml}$ of activated authologous PRP per affected knee in two applications, with an interval of 15 days. Group 2 was given celecoxib $200 \mathrm{mg}$ each $24 \mathrm{~h}$ for 1 year, irrespectively of the presence of pain; compliance was ensured with monthly appointments for the renewal of the prescription. An evaluation was performed before the treatment, and at 1, 3, 6 and 12 months after the treatment by using the WOMAC and VAS scales (Barber-Westin and Noyes, 2017). Diagnosis was performed according to the diagnostic criteria from the American College of Rheumatology (Altman et al., 1986). Inclusion criteria were knee OA grade II and III of the Kellgren-Lawrence classification; exclusion criteria were systemic pathologies as uncontrolled diabetes mellitus, rheumatoid arthtitis, axial deviation (varus $>5^{\circ}$, valgus $>9^{\circ}$ ), hematologic disorders (coagulopathies), cardiovascular diseases, infection, immunosupression, patients with anticoagulant treatment or antiplatelet agents, and alergy to celecoxib. Written informed consent was obtained from all patients. This study was approved by the Institutional Review Board of the Hospital (study number: R-2016-2001-8) and it was performed in compliance with the norms of the Mexican Health Bureau and according to the guidelines of the Declaration of Helsinki.

\section{PRP treatment}

PRP is an autologous concentration of platelets; Platelet-rich plasma without leukocytes was used in the present study. $\mathrm{Pa}-$ tients in group 1 were only given PRP; No NSAID or steroidal drug was given to them. Patients were instructed to fast over 8 hours and to avoid any NSAIDs or any steroidal drugs 72 hours before the collection of the blood sample. Sample was prepared according to Anitua Aldecoa and Andía Ortiz (2000), as follows: For each knee with OA, $10 \mathrm{ml}$ of peripheral blood were obtained; after, it was centrifuged at $1800 \mathrm{rpm}$ for $8 \mathrm{~min}$ utes, obtaining $3 \mathrm{ml}$ of PRP; afterwards, $300 \mu \mathrm{l}$ of $10 \%$ calcium chloride were added as activator. $3 \mathrm{ml}$ of activated PRP were administered intra-articularly in each affected knee, in asceptic conditions. A one-time reinjection of PRP from a fresh blood sample was performed 15 days after. After each injection, patients were allowed full weight bearing with the affected knee and instructed to apply ice for $20 \mathrm{~min}$ each $6 \mathrm{~h}$ for $24 \mathrm{~h}$ and to avoid vigorous activities for $72 \mathrm{~h}$ after each injection.

\section{Statistical analysis}

Descriptive statistics and frecuencies were obtained; standard deviations and standard errors of the mean were calculated. Kolmogorov-Smirnov and Shapiro-Wilk with a $95 \%$ confidence interval, two-tailed Fisher's exact test, two-tailed Student's $t$-test and Mann-Whitney $U$ were performed when applicable. A linear regression was performed to determine an association with age. Analyses were repeated adjusting for all variables; $p<0.05$ was considered significant. Statistical analysis was performed by the use of the software SPSS from IBM, version 23 for Macintosh.

\section{Results}

A total of 60 patients with a median age of $53.25 \pm 9.0$ years were included in the study and randomly allocated in two groups of 30 patients each. The PRP group was composed by 4 males and 26 females, a mean age of $53.7 \pm 8.58$, both knees in 19 individuals; and with 13 and 17 patients with knee OA grade 2 and 3 , respectively. The celecoxib group was composed by 9 males and 21 females, a mean age of $52.8 \pm 9.64$, both knees in 18 individuals; and with 18 and 12 patients with knee OA grade 2 and 3 , respectively.

No patient abandoned the study or required another medication for the treatment of pain. At the beginning of the study, there was no significant intra- or intergroup general differences in sex $(p>0.05)$, age $(p>0.05)$, number of affected knees $(p>0.05)$, grade of arthrosis $(p>0.05)$, VAS $(p>0.05)$, total WOMAC $(p>0.05)$ or in any WOMAC subscale $(p>0.05)$ (Table 1). In addition, between the start and the end of the study there was a significant improvement on VAS in both treatments (PRP group: 68.69\%, $p<0.001$; celecoxib group: $40.94 \%, p<0.001)$. Likewise, at the end of the study, there was a significant improvement with PRP in comparison to celecoxib in final VAS ( $p<0.001,40.40 \%$ improvement), total WOMAC (58.95\%) $(p<0.001)$ and in the WOMAC subscales of pain ( $p=7.043 \times 10^{-7}, 50.60 \%$ improvement), function $\left(p=3 \times 10^{-6}, 51.90 \%\right.$ improvement $)$ and stiffness $(p<0.001$, $34.13 \%$ improvement) (Fig. 1); the significant differences persisted with PRP in comparison to celecoxib after adjusting for sex $(p<0.05)$ or for any grade of knee OA $(p<0.01)$. Furthermore, there was no significant linear correlation of age in any group on initial or final VAS, total WOMAC or in any WOMAC subscale $(p>0.05)$ (Table 2). 24-48 h pain in the site of injection was the only side effect found in the PRP group; it was only present in the first injection and managed with ice and rest. There was no side effect found in the Celecoxib group.

\section{Discussion}

The significant improvement in pain, stiffness and function with PRP in comparison to celecoxib is concordant with the results of previous studies comparing PRP against hyaluronic acid and placebo (Kavadar et al., 2015; Laudy et al., 2015; Sanchez et al., 2012; Say et al., 2013; Spakova et al., 2012; Vaquerizo et al., 2013) or etoricoxib (Buendia-Lopez et al., 2018). Moreover, our results at the end of the study, together with the lack of significant differences between both groups before treatment, suggest a higher effectiveness of PRP against NSAIDs in decreasing knee OA's associated pain and stiffness and in improving knee OA's function; which is concordand with the results recently reported by Buendía-López, 
Table 1. General characteristics of the patients at the start of the study

\begin{tabular}{lccc}
\hline Parameters & PRP group $(n=30)$ & Celecoxib group $(n=30)$ & PRP group vs Celecoxib group \\
\hline Male/Female $(n)$ & $4 / 26$ & $9 / 21$ & $p=0.209$ \\
Mean age (years) & 53.7 & 52.8 & $p=0.683$ \\
Both knees $(n)$ Yes/No & $19 / 11$ & $18 / 12$ & $p=1.000$ \\
Grade of knee OA $(n)$ 2/3 & $13 / 17$ & $18 / 12$ & $p=0.302$ \\
WOMAC & & & $p=0.301$ \\
Mean Total baseline & $15.77 \pm 4.915$ & $14.311 \pm 5.868$ & $p=0.644$ \\
Mean Pain baseline & $9.10 \pm 3.614$ & $9.40 \pm 3.847$ & $p=0.069$ \\
Mean Stiffness baseline & $3.90 \pm 1.561$ & $3.07 \pm 1.911$ & $p=0.238$ \\
Mean Function baseline & $34.30 \pm 11.271$ & $30.467 \pm 13.521$ & $p=0.988$ \\
Mean VAS baseline & $7.57 \pm 1.675$ & $7.67 \pm 1.749$ & \\
\hline
\end{tabular}
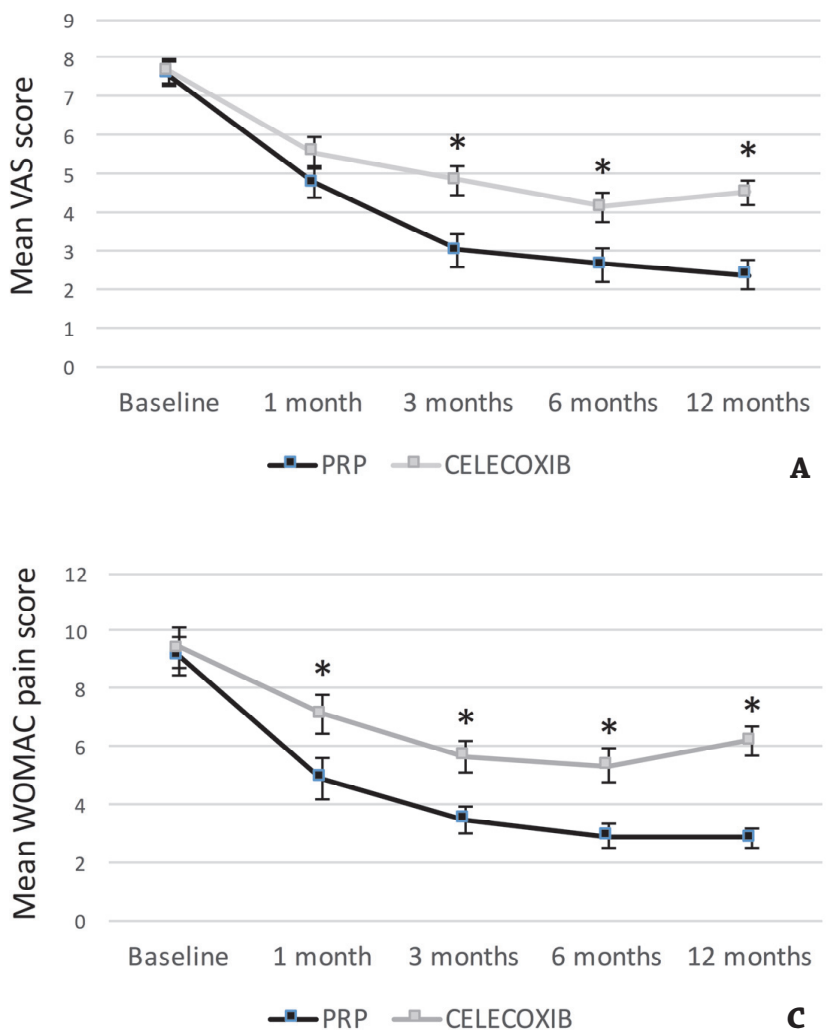
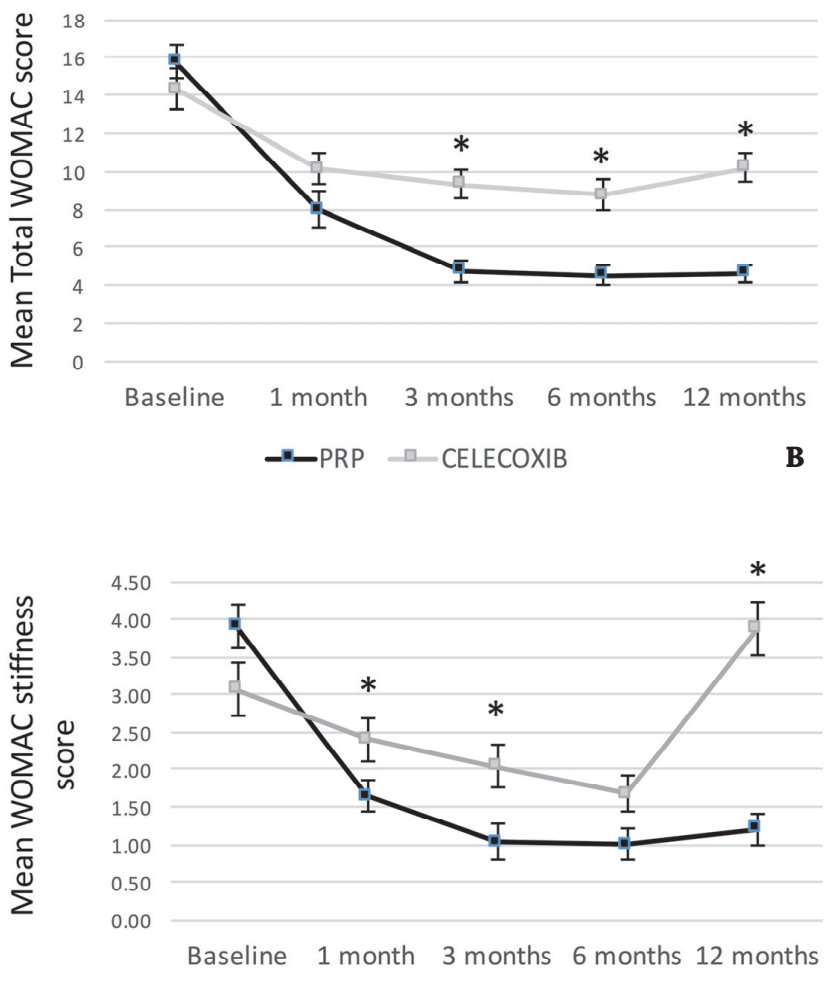

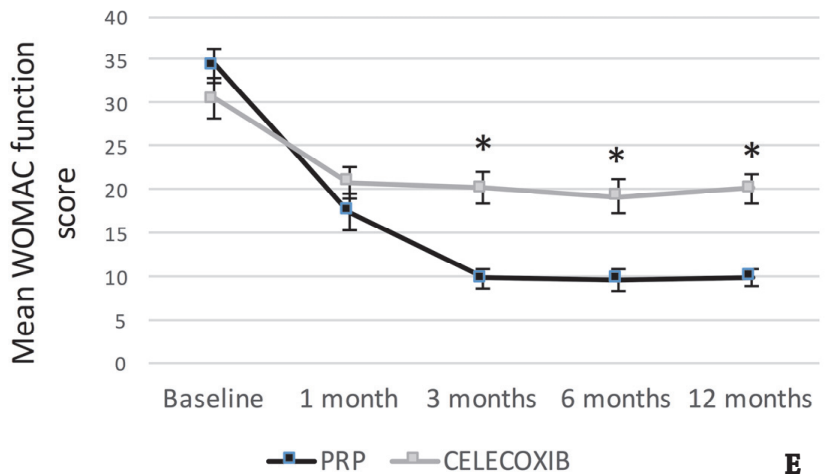

Fig. 1. Chronological comparison of results of PRP and celecoxib.

The results for (A) VAS, (B) total WOMAC and (C) WOMAC pain, (D) stiffness and (E) function at baseline and at 1, 3, 6 and 12 months after the start of the study. Error bars indicate S.E.M. ${ }^{*} p<0.05$. 
Table 2. Correlation of parameters with age

\begin{tabular}{lcc}
\hline Parameters & $\begin{array}{c}\text { Correlation } \\
\text { with age in the } \\
\text { PRP group }\end{array}$ & $\begin{array}{c}\text { Correlation } \\
\text { with age in the } \\
\text { celecoxib group }\end{array}$ \\
\hline WOMAC & $p=0.306$ & $p=0.408$ \\
Baseline total & $p=0.093$ & $p=0.993$ \\
Baseline pain & $p=0.619$ & $p=0.291$ \\
Baseline rigidity & $p=0.464$ & $p=0.283$ \\
Baseline functional capacity & $p=0.582$ & $p=0.813$ \\
Final total & $p=0.600$ & $p=0.427$ \\
Final pain & $p=0.145$ & $p=0.265$ \\
Final rigidity & $p=0.815$ & $p=0.581$ \\
Final functional capacity & & \\
VAS & $p=0.325$ & $p=0.601$ \\
Baseline & $p=0.402$ & $p=0.510$ \\
Final & &
\end{tabular}

There was no correlation of any of the measured parameters with age.

et al. (2018). In addition, as previously reported in other studies (Kon et al., 2010; Patel et al., 2013; Pourcho et al., 2014), age or sex were not correlated with PRP treatment's outcome. In this respect, Bottegoni et al. (2016) found a significant decreased effect of PRP in patients of 80 years of age or older, however there was not possible for us to study that variable since there was no patient in our study of that age or older. Likewise, several previous studies found that the lower the degree of knee OA, the better the response to PRP (Bottegoni et al., 2016; Montanez-Heredia et al., 2016; Pourcho et al., 2014); however, in our study this was only the case for PRP in final AVS and in the final WOMAC subscales of pain and functional capacity. In this respect, our results also suggest that despite PRP is more effective at lower knee OA degrees, as previously reported by Kilincoglu et al. (2015), there is also a significant beneficial effect in patients with higher knee OA degrees, which is also concordant with the results found by Kavadar et al. (2015). Therefore, our results support the findings of a previous meta-analysis, where a benefit, despite with limited to moderate evidence, was found in favour of PRP against placebo, controls and hyaluronic acid (Laudy et al., 2015). Due to the serious side effects of NSAIDs or steroids, the use of other effective therapies would be a clinical advantage in the treatment of knee OA. In that matter, PRP has been found to have no significant differences in local or systemic side effects in comparison to placebo (Laudy et al., 2015). Future studies will determine if the improvement of PRP in knee OA is still significant when used in more advanced grades of knee OA and will characterize the molecular mechanism of action of PRP and the specific molecules contained in it that are responsible for its beneficial effect in early knee OA.

\section{Conclusions}

Intra-articular PRP is significantly better than celecoxib in improving pain, function and stiffness in early knee OA. This significant difference is present irrespectively of age, sex or knee OA grade II or III.

\section{Conflict of interests}

The authors report no conflict of interests.

\section{References}

Altman R, Asch E, Bloch D, Bole G, Borenstein D, Brandt K, et al. (1986). Development of criteria for the classification and reporting of osteoarthritis. Classification of osteoarthritis of the knee. Diagnostic and Therapeutic Criteria Committee of the American Rheumatism Association. Arthritis Rheum 29(8): 1039-1049. DOI: 10.1002/art.1780290816.

Anitua Aldecoa E, Andía Ortiz I (2000). Un nuevo enfoque en la regeneración ósea. Plasma rico en factores de crecimiento (PRGF). Vitoria-España: Puesta al Día Publicaciones.

Barber-Westin SD, Noyes FR (2017). Rating of Athletic and Daily Functional Activities: Knee-Specific Scales and Global Outcome Instruments. In: Barber-Westin SD, Noyes FR (Eds). Noyes' Knee Disorders: Surgery, Rehabilitation, Clinical Outcomes (pp. 1211-1221). Philadelphia: Saunders.

Bottegoni C, Dei Giudici L, Salvemini S, Chiurazzi E, Bencivenga R, Gigante A (2016). Homologous platelet-rich plasma for the treatment of knee osteoarthritis in selected elderly patients: an open-label, uncontrolled, pilot study. Ther Adv Musculoskelet Dis 8(2): 35-41. DOI: 10.1177/1759720X16631188.

Buendia-Lopez D, Medina-Quiros M, Fernandez-Villacanas Marin MA (2018). Clinical and radiographic comparison of a single LP-PRP injection, a single hyaluronic acid injection and daily NSAID administration with a 52-week follow-up: a randomized controlled trial. J Orthop Traumatol 19(1): 3. DOI: 10.1186/ s10195-018-0501-3.

Hochberg MC, Altman RD, Toupin April K, Benkhalti M, Guyatt G, McGowan J, et al. (2012). American College of Rheumatology 2012 Recommendations for the Use of Nonpharmacologic and Pharmacologic Therapies in Osteoarthritis of the Hand, Hip, and Knee. Arthrit Care Res 64(4): 465-474. DOI: 10.1002/acr.21596.

Kavadar G, Demircioglu DT, Celik MY, Emre TY (2015). Effectiveness of platelet-rich plasma in the treatment of moderate knee osteoarthritis: a randomized prospective study. J Phys Ther Sci 27(12): 3863-3867. DOI: 10.1589/jpts.27.3863.

Kilincoglu V, Yeter A, Servet E, Kangal M, Yildirim M (2015). Short term results comparison of intraarticular platelet-rich plasma (prp) and hyaluronic acid (ha) applications in early stage of knee osteoarthritis. Int J Clin Exp Med 8(10): 18807-18812.

Kon E, Buda R, Filardo G, Di Martino A, Timoncini A, Cenacchi A, et al. (2010). Platelet-rich plasma: intra-articular knee injections produced favorable results on degenerative cartilage lesions. Knee Surg Sports Traumatol Arthrosc 18(4): 472-479. DOI: 10.1007/ s00167-009-0940-8.

Laudy ABM, Bakker EWP, Rekers M, Moen MH (2015). Efficacy of platelet-rich plasma injections in osteoarthritis of the knee: a systematic review and meta-analysis. Br J Sports Med 49(10): 657-672. DOI: 10.1136/bjsports-2014-094036.

Law TY, Nguyen C, Frank RM, Rosas S, McCormick F (2015). Current concepts on the use of corticosteroid injections for knee osteoarthritis. Phys Sportsmed 43(3): 269-273. DOI: 10.1080/00913847.2015.1017440.

Lee T, Lu N, Felson DT, Hyon KC, Dalal DS, Zhang Y, Dubreuil M (2016). Use of non-steroidal anti-inflammatory drugs correlates with the risk of venous thromboembolism in knee osteoarthritis patients: a UK population-based case-control study. Rheumatology (Oxford) 55(6): 1099-1105. DOI: 10.1093/rheumatology/kew036.

Montanez-Heredia E, Irizar S, Huertas PJ, Otero E, Del Valle M, Prat I, et al. (2016). Intra-Articular Injections of Platelet-Rich Plasma versus Hyaluronic Acid in the Treatment of Osteoarthritic Knee Pain: A Randomized Clinical Trial in the Context of the Spanish National Health Care System. Int J Mol Sci 17(7): 1064. DOI: 10.3390/ijms17071064.

Mundy L (2016). Platelet-rich plasma: a case study for the identification of disinvestment opportunities using horizon scanning. Aust Health Rev 41(1). DOI: 10.1071/AH15075.

Patel S, Dhillon MS, Aggarwal S, Marwaha N, Jain A (2013). Treatment With Platelet-Rich Plasma Is More Effective Than Placebo for Knee Osteoarthritis. A Prospective, DoubleBlind, Randomized Trial. Am J Sports Med 41(2): 356-364. DOI: $10.1177 / 0363546512471299$. 
Pourcho AM, Smith J, Wisniewski SJ, Sellon JL (2014). Intraarticular platelet-rich plasma injection in the treatment of knee osteoarthritis: review and recommendations. Am J Phys Med Rehabil 93(11 Suppl. 3): S108-121. DOI: 10.1097/ PHM.0000000000000115.

Sakata R, McNary SM, Miyatake K, Lee CA, Van den Bogaerde JM, Marder RA, Reddi AH (2015). Stimulation of the superficial zone protein and lubrication in the articular cartilage by human platelet-rich plasma. Am J Sports Med 43(6): 1467-1473. DOI: $10.1177 / 0363546515575023$

Sanchez M, Fiz N, Azofra J, Usabiaga J, Aduriz Recalde E, Garcia Gutierrez A, et al. (2012). A randomized clinical trial evaluating plasma rich in growth factors (PRGF-Endoret) versus hyaluronic acid in the short-term treatment of symptomatic knee osteoarthritis. Arthroscopy 28(8): 1070-1078. DOI: 10.1016/j. arthro.2012.05.011.

Say F, Gürier D, Yener K, Bülbül M, Maikoç M (2013). Platelet-Rich Plasma Injection Is More Effective than Hyaluronic Acid in the Treatment of Knee Osteoarthritis. Acta Chir Orthop Traumatol Cech 80(4): 278-283.

Simon LS (2013). Nonsteroidal anti-inflammatory drugs and their risk: a story still in development. Arthritis Res Ther 15(Suppl. 3): S1. DOI: 10.1186/ar4173.

Solomon DH, Husni ME, Libby PA, Yeomans ND, Lincoff AM, Lupsilonscher TF (2017). The Risk of Major NSAID Toxicity with Celecoxib, Ibuprofen, or Naproxen: A Secondary Analysis of the PRECISION Trial. Am J Med 130(12): 1415-1422.e4. DOI: 10.1016/j.amjmed.2017.06.028.
Spakova T, Rosocha J, Lacko M, Harvanova D, Gharaibeh A (2012). Treatment of knee joint osteoarthritis with autologous plateletrich plasma in comparison with hyaluronic acid. Am J Phys Med Rehabil 91(5): 411-417. DOI: 10.1097/PHM.0b013e3182aab72.

Sundman EA, Cole BJ, Karas V, Della Valle C, Tetreault MW, Mohammed HO, Fortier LA (2014). The anti-inflammatory and matrix restorative mechanisms of platelet-rich plasma in osteoarthritis. Am J Sports Med 42(1): 35-41. DOI: $10.1177 / 0363546513507766$

Vaquerizo V, Plasencia MA, Arribas I, Seijas R, Padilla S, Orive G, Anitua E (2013). Comparison of intra-articular injections of plasma rich in growth factors (PRGF-Endoret) versus Durolane hyaluronic acid in the treatment of patients with symptomatic osteoarthritis: a randomized controlled trial. Arthroscopy 29(10): 1635-1643. DOI: 10.1016/j.arthro.2013.07.264.

Wang CC, Lee CH, Peng YJ, Salter DM, Lee HS (2015). PlateletRich Plasma Attenuates 30-kDa Fibronectin Fragment-Induced Chemokine and Matrix Metalloproteinase Expression by Meniscocytes and Articular Chondrocytes. Am J Sports Med 43(10): 2481-2489. DOI: 10.1177/0363546515597489.

Yin WJ, Xu HT, Sheng JG, An ZQ, Guo SC, Xie XT, Zhang CQ (2016). Advantages of Pure Platelet-Rich Plasma Compared with Leukocyte- and Platelet-Rich Plasma in Treating Rabbit Knee Osteoarthritis. Med Sci Monit 22: 1280-1290. DOI: 10.12659/ msm.898218. 